\title{
BIODETERIORO DE MATERIALES PLÁSTICOS POR MICROHONGOS
}

Palabras clave: Plásticos, polímeros, biodeterioro, hongos.

Keys words: Plastics, polymers, biodeterioration, fungi.

\section{RESUMEN}

Mediante la selección de 8 compuestos plásticos utilizados en el mercado, adquiridos a la firma Aldrich Quimica S.R.L. (Alemania), se valoró su capacidad de ser asimilados como única fuente de carbono in vitro, por 2 grupos de microhongos pertenecientes a los AscoDeuteromycetes. Estos compuestos fueron: poliestireno, polivinilacetato, poliacrilonitrito, policaprolactona, polimetacrilato de metilo, polivinilclontro, acetato de celulosa y polietileno.

Solo la policaprolactona y el polivinilacetato fueron utilizados por los 2 grupos de hongos en estudio, en especial por cepas de Aspergillus, Chaetomium, Cladosporium, Fusarium, Penicillium y Scopulariopsis. La policaprolactona fue el polimero más utilizado entre pH 6,5 a 8 .

Las diferentes especies pertenecientes a los géneros Aspergillus y Fusarium, presentaron la mayor actividad en la utilización de estos compuestos.

\section{INTRODUCCION}

Los hongos como heterótrofos, desempeñan conocidas actividades en la descomposición de una gran variedad de susbtancias orgánicas y en la oxidación de algunos compuestos inorgánicos en la biosfera, especialmente en su rol de biotrofos.

Los plásticos en general han tenido una enorme difusión comercial en los últimos decenios, gracias a su alta resistencia a la descomposición y a las posibilidades industriales de sintesis de nuevos polímeros con propiedades fisico-quimicas especiales. Paralelamente a estas cualidades, responsables de sus éxitos, se han presentado problemas graves en el campo de los deshechos, debido al limitado o nulo ataque biológico hacia ellos por parte de los microorganismos. Esta situación ha permitido que los

\section{Anna Maria Mangiarotti*, Giuseppe Caretta, * Enrico Nelli* \& Edoardo Piontelli** *Universitá Degli Studi Di Pavia Istituto di Micologia Medica " R Ciferri e P. Redaelli" Via San Epifanio 14. Pavia (Italia). \\ **Universidad de Valparaiso, Escuela de Medicina Cátedra de Micología, Casilla 92 V, Valparaiso (Chile).}

\section{SUMMARY}

[Biodeterioration of plastic comppounds by microfungi]

By means of the selection of 8 plastic compounds that are found in the industry, and bought from Aldrich Chemical S.R.L.(Germany), the capacity to be assimilated as the only carbon source in vitro, by two groups of microfungi belonging to Asco-Deuteromycetes, was evaluated. These materials were:Polystyrene,polyvinylacetate, polvacrylonitrite, polycaprolactone, polymethylmethacrylate, polyvinylchlorure, cellulose acetate and polyethylene.

Only polycaprolactone and polyvinylacetate were used by both groups of fungi under study, specially by strains of Aspergillus, Chaetomium, Cladosporium, Fusarium, Penicillium and Scopulariopsis. Polycaprolactone was the polymer most utilized between $\mathrm{pH}$ 6,5 to 8 .

The different species belonging to genera Aspergillus and Fusarium, presented the highest activity in the utilization of these compounds.

productos manufacturados con plásticos, permanezcan visibles en muchos ambientes terrestres o aruáticos, debido a su difícil eliminación (Eggins et al., 1971,1975).

Quizás la solución actual, reside en las posibilidades de un total o parcial reciclaje industrial, para evitar que la incineración, al producir substancias tóxicas (ac. clorhidrico, dioxinas, etc), produzca graves daños biológicos a un sinnúmero de organismos (Palit \& Bhattacharya 1976).

Con el advenimiento de plásticos foto y biodegradables, se pensó en haber logrado soluciones definitivas, sin embargo estas substancias han demostrado, grandes limitaciones en este campo (Klemchuk, 1989).

Entre los primeros estudios sobre la posibilidad de alterar los plásticos mediante su entierro prolongado, Conally (1971) y Kestel'man et al. (1972), descubrieron que después de varios años de permanencia bajo el suelo, se 
después de varios años de permanencia bajo el suelo, se presentaban cambios físicos, concluyendo que éstos podrian también deberse a la acción de agentes biológicos. Por el contrario, en experimentos efectuados por Rodriguez, (1971) y Potts et al.(1972), con plásticos vinilicos tales como: polietileno, polipropileno, poliestireno, polivinilcloruro y poliesteres aromáticos, se demostró su resistencia al ataque biológico.

Solo algunos tipos de plásticos (poliesteres alifáticos y poliuretanos), parecen ser los únicos polímeros capaces de ser atacados por microorganismos (Potts et al, 1972; Tokiwa \& Suszuki, 1974; Diamond et al., 1975; Klemchuk, 1989).

El poliestireno no es sensible a la degradación biológica, aunque sea recubierto por los hongos (Heap \& Morrel, 1968).

En general los estudios sobre la descomposición fúngica de los plásticos son escasos y los que existen, se limitan a unos pocos compuestos relacionados con algunas especies de hongos( Hazeau, 1967; Heap \& Morrel, 1968; Nykrist, 1974; Kavelman \& Kendrick, 1978).

Nuestro objetivo principal fue: valorar in vitro la capacidad de diferentes microhongos (Asco-Deuteromycetes), capaces de asimilar como única fuente de carbono, las diversas cadenas de polimeros de los principàles grupos de plásticos utilizadas en el comercio. Como objetivos especificos se valoraron: el crecimiento de las cepas, la importancia del $\mathrm{pH}$, las variaciones de éste, las capacidades asimilativas entre especies de un mismo género y los cambios en el aspecto físico de los polimeros utilizados.

\section{MATERIALES YMETODOS}

\section{a) Selección de los materiales}

La seleccion de los principales grupos de polimeros a utilizarse, se basó en los más empleados en el mercado y en algunos materiales plásticos usados por otros investigadores. Los compuestos de base* fueron adquiridos a la firma Aldrich Quimica S.R.L. (Alemania), presentándose ya sea bajo forma de: polvo, granos o pellet.

\footnotetext{
* Polivinil acetato(PVC) 18.949-9 (Código)

Poliestireno 18,242-7

Poliacrilonitrilo $18,131-5$

Policaprolactona $18,16()-9)$

Polimetacrilatode metilo 18,223-0

Polivinilcloruro $18,958-8$

Acetato de celulosa $18,095-5$

Polietileno 18,189-7
}

\section{b) Medio dle cultivo}

Para el control de la actividad descomponedora de lo hongos seleccionados, se adoptó la metodologia de cultivo empleada por Kavelman \& Kendrick (1978), en su actividad sobre la poli-epsilon caprolactona.

Los componentes del medio base fueron los siguientes: Nitrato de sodio, $0.3 \mathrm{~g}$; Dipotasio hidrogeno fosfato, $0,1 \mathrm{~g}$; Sulfato de magnesio, $0,5 \mathrm{~g}$; Cloruro de potasio 0,5g; Sulfato ferroso, $0.01 \mathrm{~g}$; Agar noble, $15 \mathrm{~g}$; Agua destilada, $1000 \mathrm{ml}$ (Agar Czapek-Dox).

El agar noble utilizado, fue para evitar posibles traza de microelementos nutritivos.

Para el crecimiento activo de las cepas (inóculos controles), se empleó Agar extracto de Malta a pH 7.

\section{c) Cepas fúngicas utilizadas}

Se utilizaron cepas füngicas provenientes de micoteca del "Istituto di Micología Medica" de la Universidad de Pavia (Italia), previamente seleccionada por su capacidad celulolitica y que evidenciaron resultados positivos en ensayos previos, frente a policaprolactona

\section{Grupo 1.}

A. niger van Tieghem 1

A.niger 3 .

'A.ustus (Bain.) Thom \& Church

Cladosporium sphaerospermum Penz.

Chictomium globosum Kunze ex Steud.

Fusarium sp.

F.oxysporum Schlecht

Penicillium fellutanum Biourge

P.thomii Maire

P.velutinum van Beyma

Scopulariopsis brevicaulis (Sacc.)Bain.

Para evidenciar las posibles diferencias en la utilización de estos polímeros, entre especies de un mismo género, se seleccionaron 18 especies cosmopolitas, de Hyphomycetes comunes en los suelos, pertenecientes a los géneros Aspergillus, Fusarium y Penicillium (6 diferentes para cada género).Esta prueba no incluyó ninguna de las especies del Grupo 1.

\section{Grupo 2.}

Aspergillus glaucus Link

A. funiggatus Fres.

A.versicolor (Vuill.) Tirahoschi

A flavus Link

A.nidulellus Samson \& W.Gams (= A.nidulans)

A.alutaceus Berk. (=A.ochraceus Wilhelm)

Fusarium oxysporum Schlecht. emend. Snyd \&

Hans.

F.merismoides Corda 
F.lateritium Nees

F.semitectum Berk. \& Rav.

F.equiseti ( Corda) Sacc.

F.tricinctum (Corda) Sacc.

Penicillium jantinellum Biourge

P.purpurogenum Stoll

P.funiculosum Thom

P.glabrum (Wehmer) Westling (= P.frecuentans)

P.expansum Link

P.brevicompactum Dierchx

Todas las cepas del Grupo 1 y 2 , se cultivaron en MEA durante un lapso de 7 a 14 días, a $25^{\circ} \mathrm{C}$, hasta obtener una buena esporulación para el inóculo.

\section{d) Preparación del inóculo y siembras}

1) El inóculo consistió en una suspensión en $5 \mathrm{ml}$ de $\mathrm{H}_{2} \mathrm{O}$ estéril, de un raspado con asa, de la superficie de cada colonia con buena fiuctificación, hasta la obtención de una turbidez de 0,2 correspondiente a la escala McFarland.

De cada suspensión se tomó $1 \mathrm{ml}$, distribuyendose y homogenizandose en placas de Petri de $10 \mathrm{~cm}$ ( llenadas en sus $3 / 4$ en altura) con el medio mineral, licuado a $45^{\circ} \mathrm{C} . \mathrm{y}$ a diferentes $\mathrm{pH}$ finales $(4,6,5$ y 8 ). La única fuente de carbono, correspondió a cada tipo de material plástico utilizado. Como control, los hongos en estudio fueron incubados en el mismo medio mineral (sin ninguna fuente de carbono).

Los materiales plásticos en pellet y granos, se depositaron con pinzas estériles en el centro de las placas en cantidades de 5 a 8 , mientras los materiales en polvo, en la misma posición central, pero con una cuchareta de $5 \mathrm{~mm}$ de diámetro.

Todas las siembras de las cepas descritas en el Grupo

1 y los controles, fueron efectuadas en duplicado.

Las placas se incubaron por un periodo de 2 semanas a $25^{\circ} \mathrm{C}$ y a temperatura ambiente hasta completar 60 dias (entre $18-23^{\circ} \mathrm{C}$ )

Para evitar la desecación rápida del medio de cultivo, por el prolongado periodo de incubación, éstas después de las 3 semanas se envolvieron en grupos, con un delgado plástico transparente.

El crecimiento de cada colonia fue representado por una simbologia de cruces: $+/++/+++$, en función de: un crecimiento escaso (colonias de 0,1 hasta $1 \mathrm{~cm}$ de diámetro), discreto ( colonias de hasta $2 \mathrm{~cm}$ ) y bueno (colonias superiores a $2 \mathrm{~cm}$ ) respectivamente.

Para valorar el crecimiento total en cada unidad de tiempo y $/ 0 \mathrm{pH}$, se obtuvo un promedio aproximado al entero más proximo del número de cruces asignado a cada crecimiento a los 60 dias (no importando si este continuaba posteriormente). En las mediciones del diámetro de las colonias, se consideró sólo el crecimiento observado en la superficie del material o en sus proximidades, seleccionando solo los talos que permitieron una mejor medición. El crecimiento sobre los polímeros o en sus proximidades se aceptó como una prueba de la utilización de éste.

Solo se consideró como crecimiento cuantificable de una cepa, aquél que tuviera negativo ocon leve desarrollo el control correspondiente.

Como prueba anexa para apreciar la influencia del $\mathrm{pH}$ sobre el crecimiento, se cultivaron todas las cepas del Grupo 1, en un medio con compuestos carbonados de fácil utilización (Agar extracto de Malta), utilizandose como prueba (en duplicado), el cultivo de las cepas del Grupo 1.

Para apreciar las variaciones del $\mathrm{pH}$ en el tiempo (inicial ( $\mathrm{pH} 6,5), 30$ y 60 días), frente a los distintos polimeros, se utilizó el mismo medio de cultivo mineral, pero líquido, en tubos de ensayo con 9cc del medio y con 3 gramos c/u, de cada tipo de plástico. Para las mediciones se empleó un peachimetro digital Bekman. Para este ensayo, solo se empleó la cepa de mejor crecimiento de cada género (del Grupo 1).

2) Todas las cepas del Grupo 2, se estudiaron con la misma metodología estipulada en el punto d. 1, pero solamente en agar Czapek-Dox, con pH final de 6,5.

e) Cambios en el aspecto visual de los polímeros empleados.

Para apreciar los posibles cambios superficiales de los granos o pellet de polietileno empleados, después del periodo de incubación (solo se utilizaron los hongos del Grupo 1, considerados como patrones), éstos se retiraron de lasplacas, se dejaron en agua destilada estéril por 1 hora, posteriormente se lavaron cuidadosamente para retirar todo resto del micelio presente ( $\sin$ emplear elementos que permitieran dañar su superficie). Luego se compararon con el producto original comercial, bajo la lupa estereoscòpica. Se consideró:

a) Cambios en la opacidad o brillo del material

b) Fisuras o grietas

c) Ausencia de cambios

\section{RESULTADOS}

De los 8 tipos de polímeros plásticos ensayados, solo hubo desarrollo fúngico en presencia de policaprolactona y polivinilacetato, al ser empleados éstos como única 
fuente de carbono para los dos grupos de hongos detallados en materiales y métodos. Los otros 6 tipos de polímeros analizados, arrojaron siempre resultados negativos.

\section{1) Crecimiento fúngico}

La policaprolactona, fue el polímero más utilizado por la mayoría de las especies del Grupol(Fig. 1, 2,3,4), en especial por Fusarium oxysporum, Penicillium thomii y P. fellutanum, los cuales obtuvieron los mayores diámetros en el desarrollo de sus talos. El polivinilacetato, fue utilizado en menor medida y solamente Aspergillus niger $3, F$. oxysporum y Penicillium velutinum, presentaron un escaso a moderado desarrollo (Tabla 1 ).

Solo 2 cepas del Grupo 1 ( $A$. niger $3 y F$. oxpsporum), fueron capaces de crecer en ambos polimeros.

2) Influencia del $\mathrm{pH}$

En la misma Tabla 1, puede observarse que: a) La policaprolactona permitió un mejor desarrollo de algunas cepas a un $\mathrm{pH}$ levemente ácido (cercano al neutro) o alcalino, que en $\mathrm{pH}$ ácido. En las placas con alta ácidez ( $\mathrm{pH} 4)$, solo hubo un débil desarrollo de Aspergillus ustus y Chaetomium globosum. b) Las cepas en polivinilacetato, no evidenciaron cambios mayores entre $\mathrm{pH} 6,5$ y 8, y no hubo desarrollo a pH 4 (Tabla 1).

Sobre agar extracto de Malta, en general se evidenció la capacidad de todas las cepas del $\mathbb{G}$ rupo 1 , de crecer tanto a $\mathrm{pH} 4$ como a pH 8, aunque entre $\mathrm{pH} 6,5$ y 8 , los diámetros fueron ligeramente superiores en promedio.

3) Variaciones del $p H$

En presencia de policaprolactona, las variaciones de $\mathrm{pH}$ registradas en el medio líquido (Tabla 2), ponen en evidencia una ligera reducción hacia la acidez, no superio a un dígito (como máximo de pH 6,5 a 5,5).

4) Capacidades asimilativas entre especies de uI mismo género

En las especies del Grupo 2, la policaprolactona fu también el polímero más utilizado, siendo las especies d Aspergillus y Fusarium las que presentaron mayo desarrollo de sus talos, mientras que en las del géner Penicillium, sus respuestas fueron escasas, a excepció de P.funiculosum. En polivinilacetato, tanto las especie de Aspergillus como las de Fusarium tuvieron res puestas similares y superiores a las de Penicillium, excepción de P.funiculosum, todas las demás especie consideradas de este género arrojaron resultado negativos(Tabla 3 ).

5) Cambios en el aspecto físico de los polimeros

Después de 60 días de incubación y debido crecimiento fúngico en presencia de los 2 polímeros, esto compuestos presentaron al exámen visual comparativ bajo la lupa, modificaciones en brillo y transparencia Todos los hongos del Grupo 1 (grupo patrón),capaces d utilizar estos polimero, causaron una notoria opacidad er ellos, pero además $A$.niger 1 , Chaetomium globosumy $P$. thomii, produjeron ligeras fisuras y surcos en la superfici de la policaprolactona.

TABLA 1. Cepas patrones del Grupo $1 \mathrm{en}$ medio mineral sólido con los polímeros utilizados

\begin{tabular}{|c|c|c|c|c|c|c|c|c|c|}
\hline \multirow{2}{*}{$\begin{array}{l}\text { Nombre de los polímeros } \\
\text { Nombre de las cepas }\end{array}$} & \multicolumn{3}{|c|}{$\frac{\text { Polivinilacetato }}{\mathrm{pH}}$} & \multicolumn{3}{|c|}{$\frac{\text { Policaprolactona }}{\mathrm{pH}}$} & \multicolumn{3}{|c|}{$\frac{\text { Otros polímeros* }}{\mathrm{pH}}$} \\
\hline & 4 & 6,5 & 8 & 4 & 6,5 & 8 & 4 & 6,5 & 8 \\
\hline Aspergillus niger 1 & - & - & - & - & $H$ & $H+$ & - & - & - \\
\hline A.niger 3 & - & + & + & - & + & + & - & - & - \\
\hline A. ustus & - & - & - & + & $H$ & $H$ & - & - & - \\
\hline Chaetomizm globosum & - & - & - & + & $H$ & $+H$ & - & - & - \\
\hline Cladosporium sphaerospermum & - & - & - & - & $H$ & $H$ & - & - & - \\
\hline Fusarium oxysporum & - & $H$ & $H$ & - & $H+$ & $H$ & - & - & - \\
\hline Fusarium sp.1 & - & - & - & - & $H$ & $H$ & - & - & - \\
\hline Penicillium fellutanum & - & - & - & - & $+H$ & $H+$ & - & - & - \\
\hline P. thomaii & - & - & - & - & $H$ & $H+$ & - & - & - \\
\hline P. velstinums & - & $H$ & $H$ & - & - & - & - & - & - \\
\hline Scopulariopsis brevicaulis & - & - & - & - & $H$ & $H$ & - & - & - \\
\hline
\end{tabular}

* Poliestireno, Poliacrilonitrilo, Polimetacrilato de metilo, Polivinilcloruro, Acetato de celulosa; Polietileno 
Tabla 2. Modificaciones del $\mathrm{pH}$ en el tiempo, por representantes de cada género del

Grupo 1 , en medio mineral líquido con polivinilacetato y policaprolactona

\begin{tabular}{|l|c|c|c|c|c|c|}
\hline Nombre de las cepas & \multicolumn{3}{|c|}{$\begin{array}{c}\text { Polivimilacetato } \\
\text { HH }\end{array}$} & \multicolumn{4}{|c|}{$\begin{array}{c}\text { Policaprolactona } \\
\text { pH }\end{array}$} \\
& iniciall & 30 días & 60 días & inicial & 30 días & 60 días \\
\hline Aspergillus niger 1 & 6,5 & 6,4 & 6,4 & 6,5 & 6,1 & 6,1 \\
Chaetomium globosum & - & - & - & 6,5 & 5,8 & 5,8 \\
Fusarium sp. 1 & 6,5 & 6,7 & 6,7 & 6,5 & 6,1 & 5,8 \\
Penicillium thomii & - & - & - & 6,5 & 6,1 & 6,1 \\
Scopulariopsis brevicaulis & - & - & - & 6,5 & 5,8 & 5,5 \\
\hline
\end{tabular}

Tabla 3. Especies de Aspergillus, Fusarium y Penicillium del Grupo 2, en medio mineral sólido a pH 6.5, con los polímeros utilizados.

\begin{tabular}{|l|c|c|c|}
\hline Nombre de las cepas & Pollivinil. & Policapro. & Otros $^{*}$ \\
\hline Aspergillus alutaceus & - & + & - \\
A.flavus & + & + & - \\
A.fumigatus & - & ++ & - \\
A.glaucus & + & ++ & - \\
A.nidulellus & - & ++ & - \\
A.versicolor & ++ & ++ & - \\
\hline Fusarium equiseti & + & ++ & - \\
F.lateritium & + & ++ & - \\
F.merismoides & - & ++ & - \\
F.oxysporum & + & ++ & - \\
F.semitectum & + & ++ & - \\
F.tricinctum & - & ++ & - \\
\hline Penicillium brevicompactum & - & - & - \\
P.expansum & - & - & - \\
P.glabrum & - & - & - \\
P.janthinellum & - & + & - \\
P.funiculosum & + & ++ & - \\
P.purpurogenum & - & ++ & - \\
\hline
\end{tabular}

* Poliestireno, Poliacrilonitrilo, Polimetacrilato de metilo, Polivinilcloruro, Acetato de celulosa,Polietileno. 


\section{DISCUSION}

Nuestra investigación a pesar que modificó la técnica interpretativa de Kavelman \& Kendrick (1978), referente a la utilización de la policaprolactona como única fuente de carbono para algunos Hyphomycetes, nos parece simple y confirma la utilización enzimática de este polímero por el hongo. A diferencia de estos autores, no efectuamos medidas sobre las pérdidas de peso de estos compuestos, porque, el desarrollo del talo se limitaba a un período relativamente corto, el crecimiento era solo superficial sobre un material relativamente grueso (granos) y por lo tanto no podía incidir de una manera relevante sobre la masa del polimero. Tampoco se hicieron pruebas para revelar la actividad sinérgica de algunas cepas inoculadas al mismo tiempo frente a un mismo sustrato. Por el contrario, simples valoraciones oculares del desarrollo omediciones de las variaciones de las propiedades ópticas o mecánicas, efectuadas por Hazeu (1967), guardan relación con el desarrollo del hongo, pero no demuestran la utilización del polimero.

Varios investigadores consideraron en sus estudios un solo polímero, como el poliestireno (Heap \& Morrel, 1968), la policaprolactona (Kavelman\& Kendrich, 1978), el triacrilonitrilo (Asanoet al., 1980), oel polimetilmetacrilato (Kestel'man et al.,1972). En nuestra investigación seleccionamos estos compuestos basandonos en su uso en la industria actual y en su constitución ( $99 \%$ por el polimero puro), sin el agregado de aditivos o plastificantes, los cuales en muchos casos aumentan la biodescomposición del material, como fue revelado por Hazeu (1967), para el polivinilcloruro. Debido a esto, Klausmeier \& Jones (1961), hicieron experiencias encaminadas a confirmar que estas sustancias son facilmente utilizables por los hongos, mientras Mills \& Eggins (1974), demostraron la capacidad de algunos hongos termofilicos como Aspergillus fumigatus y Sporotrichum thermophile, de utilizar la mayoría de los 30 plastificantes empleados y ser resistentes al ataque de los hongos mesófilos.

Kavelman \& Kendrich (1978), Mills \&Eggins(1974), Assano et al., (1980), utilizaron cepas aisladas desde el suelo enriquecido previamente con el polimero o sus precursores, por el contrario nosotros, utilizamos cepas de una micoteca que solo tienen en común la capacidad de descomponer en la naturaleza los polimeros naturales, como la celulosa (Domshet al., 1980). Sin embargonuestros resultados concuerdan con los primeros autores mencionados, en que la policaprolactona, un poliester alifático obtenido por polimerización de epsilon caprolactona, demostró ser el compuesto más biodegradable, principalmente por especies de Aspergillus, Chaetomium, Fusarium, Penicillium y Scopulariopsis.
Estasituación fue confirmada también por Diamond et al.(1975), Pottset al.(1972)y Klemchuc (1989), los cuales sostienen que, los polieteres alifáticos, son unos de los pocos polímeros que pueden biodegradarse lentamente

Contrariamente a los resultados obtenidos por Kaplan et al., (1979) y Kestel'man et al., (1972), no observamosel biodeterioro del polimetilmetacrilato, mientras el polivinilacetato que no había sido usado en estas pruebas demostró ser sensible al ataque de algunas especies de Aspergillus, Fusarim y Penicillium.

A pesar que nuestros resultados solo pretendieron evaluar ciertas capacidades biodescomponedoras después de evidenciar resultados positivos, nuestr investigación se orientó a los géneros de mayor actividad para establecer si estas cualidades son comunes a toda las especies de un mismo género, o más aún, a alguna cepas en el ámbito de la misma especie. En este sentid Aspergillus y Fusarium tuvieron un comportamient análogo, no asi Penicillium.

En la utilización de cepas de diversa proveniencia pero pertenecientes a la misma especie, hubieron diferencias apreciables, desde positivas a negativas, frente a los compuestos. Especialmente para algunas cepas de A.niger.

Particular atención merece la influencia del $\mathrm{pH}$ e relación al crecimiento y a la capacidad biodescomponedora sobre los plásticos. La falta de crecimiento a $\mathrm{pH} 4$, de todas las cepas sobre policaprolactona, indica el rol desfavora. ble de éste en el desarrollo del talo, ya sea inhibiendo I actividad enzimática o creando condiciones no favorable para la agresion del sustrato. Esta situación podriamos extrapolarla a la baja de $\mathrm{pH}$ observada en medio liquido sobre la policaprolactona, la cual podría ser una de las causas de la detención del crecimiento que observamo: entre los 60 y 90 días en medio sólido.

La capacidad de crecimiento fúngico bajo condiciones de laboratorio, no involucra situaciones limitantes d competencia. Bajo éstas condiciones experimentales, $s$ actividad fisiológica se centra principalmente en los facto res que puedan afectar el crecimiento de los ápices hifales y por ende la extensión del micelio. La dinámica de crecl miento impuesta por nuestras condiciones ambientale y complejos recursos nutricionales carbonados, limitaron ciertamente el crecimiento exponencial activo de las colo. nias. Tratamos de suplir la deshidratación, agregando mayor cantidad de medio de cultivo en las placas de Petri pero: la disminución de nutrientes, el acúmulo de metabolitos secundarios, los cambios de $\mathrm{pH}$, así com otros factores, no pudieron ser regulados con nuestri metodologia, lo cual puede detener totalmente el crea: miento o limitandolo solo a áreas periféricas (anill marginal) de las colonias. En el análisis de la prolongad incubación, que efectuamos en estudios previos, pudimo 
apreciar que los talos empezaban a visualizarse desde los 15 a 30 días, pero generalmente el crecimiento exponencial se apreciaba mejor entre los 30 y 60 dias. Entre los 60 y 90 días, las colonias en general aún podían crecer lentamente, pero seguramente a expensas de su anillo marginal; es por ésto que nuestras mediciones se hicieron a los 60 días, considerando que en tiempos mayores estaríamos frente a una fase de desaceleración o autolítica.

En las placas de control sin el polímero (sin nunguna fuente de carbono), los hongos no crecian o lo hacian en forma muy reducida; consideramos esta situación como normal, debido a que algunos hongos, en especial los del género Aspergillus y Fusarium, son capaces de crecer lentamente bajo condiciones oligotróficas (Wainwright, 1988), aprovechando mínimas trazas en el sustrato o reciclando los componentes de pared - citoplasma presentes en el inóculo no viable.

\section{CONCLUSIONES}

Los polímeros plásticos de policaprolactona y polivinilacetato, pueden ser utilizados como fuente de carbono por algunos Asco-Deuteromycetes, en especial por especies de Aspergillus, Fusarium y Penicillium.

La policaprolactona fue el polímero más utilizado por la mayoría de las especies del Grupo 1 y 2.

En las condiciones experimentales in vitro empleadas, las cepas estudiadas presentaron los mejores desarrollos de sus talos a pH cercanos al neutro o alcalino (6,5-8.0).

En un análisis de la capacidad de utilización delos 2 polímeros entre las especies de un mismo género, Aspergillus y Fusarium tuvieron un comportamiento similar, mientras Penicillium exhibió las mayores diferencias.

\section{REFERENCIAS}

Asano, Y.;Ando,S.;Tani, Y.; Yamada,H. \& Ueno,T. (1980).Fungal degradation of triacrylonitrile.Agric. Biol.Chem.45:57-62

Conally, R. A. (1971). Soil burial of material and structures Biodeterioration of materials. 2: 168-178.

Diamond,M.J.;Freedman,B. \& Garibaldi,J.A.(1975). Biodegradable polyester films. Int. Biodetn. Bull. 2: 127-132

Domsch,K.H.;Gams, W. \& Anderson, T.H. (1980). Compendium of soil fungi Academic press. London.

Eggins, H.O.W; Mills,J.; Holt,A. \& Scott, G. (1971). Biodeterioration and biodegradation of synthetic polymer. In: Sykes,G. \& Skinner,F.A.(Eds.)Microbial aspects of pollution. London Academic Press pp.267-279

Eggins,H.O.W. \& Allsopp,D. (1975). Biodeterioration and bio degradation by fungi. In: Smith.I.E.\& Berry,D.R.(Eds.) The filamentous fungi. Vol.I. Amold. London. pp.301-319

Hazeu, W. (1967). Result of the first inter-laboratory experiment on biodeterioration of plastics. Int. Biodetn. Bull 3:15-19

Heap,W.M. \& Morrel, S.H .(1968). Microbial deterioration of rubber and plastics. J.Appl.Chem. 18:189-194
Kaplan, D.L.; Hartenstein. \& Sutter,J. (1979). Biodegradation of Polystyrene, poly (methyl methacrylate) and phenol formaldehyde. Applied and Environmental Microbiology. $38: 551-553$

Kavelman ,R. \& Kendrick, B. (1978). Degradation of a plastic polyepsiloncaprolactone by hỵphomycetes Mycologia. 70: 87-103

Kestel'man, V. N.; Yarovenko,V.K \& Mel'nilova,E .I. (1972). The corrosion of polymetric materials under conditions of the microbiological synthesis of enzymes. Int. Biodet. Bull.8:15-19

Klausmeier, R.E. \& Jones,W.A. (1961). Microbiological degradation of plasticisers. Dev.Ind.Microbiol. 2: 47-53

Kemchuk , P.P. (1989). Degradability II. Chemistry of plastics casts a negative vote. Modern plastics International September 1989, pp 82-85

Mills, J. \& Eggins, H.O.W. (1974). The biodeterioration of certain plasticisers by themophilic fungi. Int. Biodetn. Bull. $10: 39-44$

Nykrist, N. B. (1974). Biodegradation of low-density polyethylene. Plast. and Pol. 42: 195-199 
Palit,S.R. \& Bhattacharya, S. (1976). Plastics and environ ment Science and Culture. 42: 253-258

Potts, J.E.; Clendinning,R.A. \& Ackart,W.B.(1972). An investigations of the biodegradability of packaging plastic. Environmental Protection Technology Series.U.S. Environmental Protection Agency, Washington ,D.C.

Redriguez, F. (1971). The prospects for biodegradable plastics. Chemical Technology 409-415
Tokiwa, Y \& Suzuki,T. (1974). Degradation of polyethilene glycol adipate by a fungus. J. Ferment. Technol. Osaka 52:393-398

Wainwright, M. (1988). Metabolic diversity of fungi in relatio of growth and mineral cycling in soil- a review. Trans. Br mycol. Soc. 90:159-170 

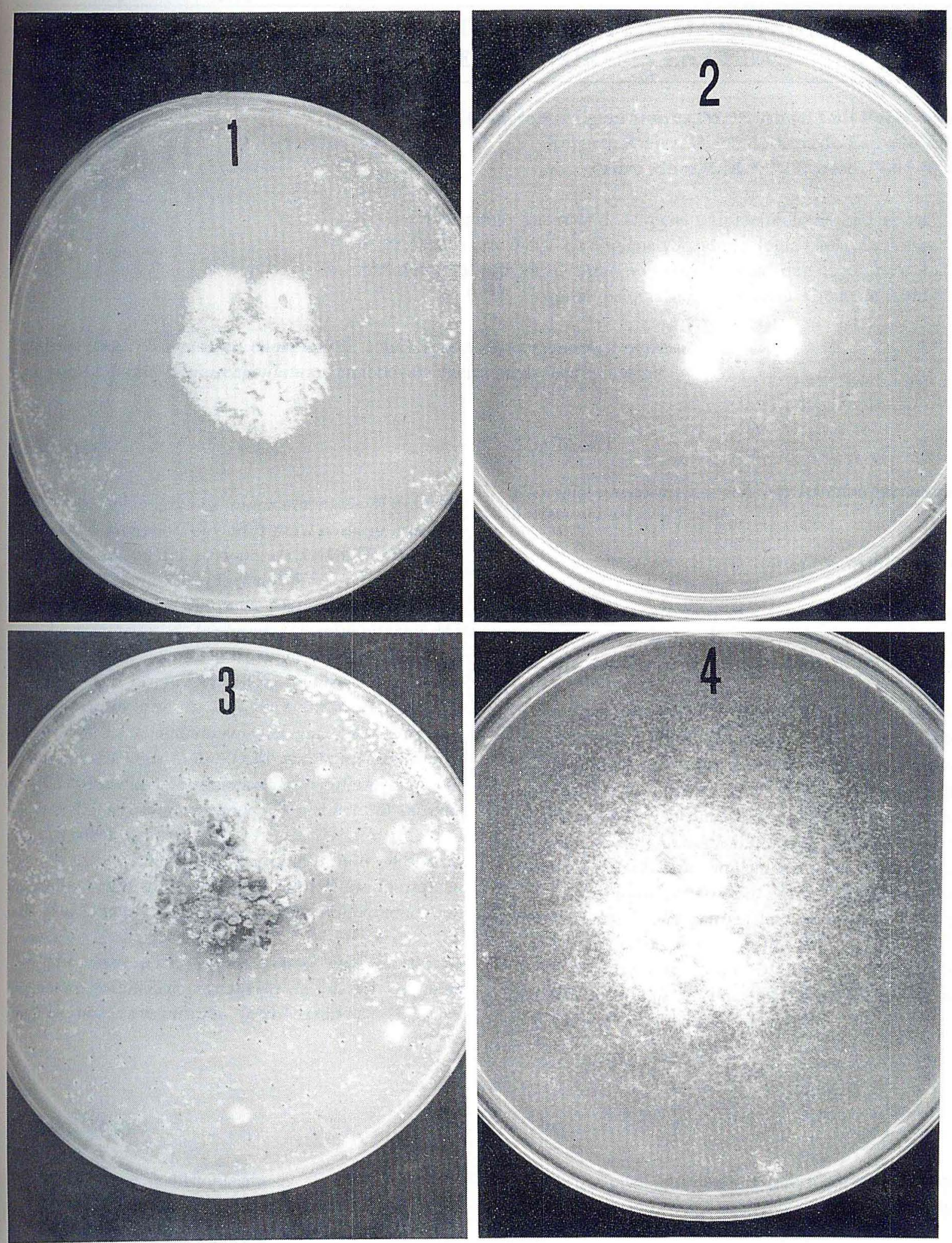

Figuras 1, 2,3,4. Medio de cultivo con policaprolactona. Fig.1. Desarrollo de Fusarium sp. . Fig.2. Desarrollo de Scopulariopsis brevicaulis . Fig 3. Desarrollo de Penicillium thomii . Fig.4. Desarrollo de Fusarium oxysporum. 